OPEN ACCESS

Edited by:

Pamela Ann McCombe, University of Queensland, Australia

Reviewed by:

Amir Sharabi,

Beth Israel Deaconess Medical Center and Harvard Medical School, United States

Emanuele D'amico,

University of Catania, Italy

${ }^{*}$ Correspondence:

Lennard Ostendorf

lennard.ostendorf@charite.de

Helena Radbruch

helena.radbruch@charite.de

Specialty section:

This article was submitted to

Multiple Sclerosis and

Neuroimmunology,

a section of the journal

Frontiers in Immunology

Received: 02 August 2019 Accepted: 06 November 2019 Published: 29 November 2019

Citation:

Ostendorf L, Mothes R, van Koppen $S$, Lindquist $R L$, Bellmann-Strobl J, Asseyer S, Ruprecht K, Alexander T, Niesner RA, Hauser AE, Paul F and Radbruch $H$ (2019) Low-Density Granulocytes Are

a Novel Immunopathological Feature in Both Multiple Sclerosis and Neuromyelitis Optica Spectrum Disorder. Front. Immunol. 10:2725. doi: 10.3389/fimmu.2019.02725

\section{Low-Density Granulocytes Are a Novel Immunopathological Feature in Both Multiple Sclerosis and Neuromyelitis Optica Spectrum Disorder}

\author{
Lennard Ostendorf ${ }^{1,2,3 *}$, Ronja Mothes ${ }^{1,4}$, Sofie van Koppen ${ }^{1,4}$, Randall L. Lindquist ${ }^{1,5}$, \\ Judith Bellmann-Strobl ${ }^{6,7}$, Susanna Asseyer ${ }^{6,8,9}$, Klemens Ruprecht ${ }^{7,10}$, Tobias Alexander ${ }^{2}$, \\ Raluca A. Niesner ${ }^{4,11}$, Anja E. Hauser ${ }^{2,3}$, Friedemann Paul ${ }^{6,7,8,10}$ and Helena Radbruch ${ }^{1 *}$
}

${ }^{1}$ Department of Neuropathology, Charité-Universitätsmedizin Berlin, Corporate Member of Freie Universität Berlin, Humboldt-Universität zu Berlin, Berlin Institute of Health, Berlin, Germany, ${ }^{2}$ Department of Rheumatology and Clinical Immunology, Charité-Universitätsmedizin Berlin, Corporate Member of Freie Universität Berlin, Humboldt-Universität zu Berlin, Berlin Institute of Health, Berlin, Germany, ${ }^{3}$ Immunodynamics, Deutsches Rheuma-Forschungszentrum Berlin, A Leibniz Institute, Berlin, Germany, ${ }^{4}$ Biophysical Analysis, Deutsches Rheuma-Forschungszentrum Berlin, A Leibniz Institute, Berlin, Germany, ${ }^{5}$ Department of Nuclear Medicine, Charité-Universitätsmedizin Berlin, Corporate Member of Freie Universität Berlin, Humboldt-Universität zu Berlin, Berlin Institute of Health, Berlin, Germany, ${ }^{6}$ NeuroCure Clinical Research Center, Charité-Universitätsmedizin Berlin, Corporate Member of Freie Universität Berlin, Humboldt-Universität zu Berlin, Berlin Institute of Health, Berlin, Germany, ${ }^{7}$ Department of Neurology, Charité-Universitätsmedizin Berlin, Corporate Member of Freie Universität Berlin, Humboldt-Universität zu Berlin, Berlin Institute of Health, Berlin, Germany, ${ }^{8}$ Experimental and Clinical Research Center, Max Delbrück Center for Molecular Medicine \& Charité-Universitätsmedizin Berlin, Corporate Member of Freie Universität Berlin, Humboldt - Universität zu Berlin, Berlin Institute of Health, Berlin, Germany, ${ }^{9}$ Department of Clinical Neurosciences, John Radcliffe Hospital, Oxford, United Kingdom, ${ }^{10}$ Clinical and Experimental Multiple Sclerosis Research Center, Charité-Universitätsmedizin Berlin, Corporate Member of Freie Universität Berlin, Humboldt-Universität zu Berlin, Berlin Institute of Health, Berlin, Germany, ${ }^{11}$ Fachbereich Veterinärmedizin, Institute of Veterinary Physiology, Freie Universität Berlin, Berlin, Germany

Objective: To investigate whether low-density granulocytes (LDGs) are an immunophenotypic feature of patients with multiple sclerosis (MS) or neuromyelitis optica spectrum disorder (NMOSD).

Methods: Blood samples were collected from 20 patients with NMOSD and 17 patients with MS, as well as from 15 patients with Systemic Lupus Erythematosus (SLE) and 23 Healthy Donors (HD). We isolated peripheral blood mononuclear cells (PBMCs) with density gradient separation and stained the cells with antibodies against CD14, CD15, CD16, and CD45, and analyzed the cells by flow cytometry or imaging flow cytometry. We defined LDGs as CD14- CD15 high and calculated their share in total PBMC leukocytes $\left(C D 45^{+}\right)$as well as the share of CD16 ${ }^{\text {hi }}$ LDGs. Clinical data on disease course, medication, and antibody status were obtained.

Results: LDGs were significantly more common in MS and NMOSD than in HDs, comparable to SLE samples (median values HD 0.2\%, MS 0.9\%, NMOSD 2.1\%, SLE $4.3 \%$ ). $0 / 23$ of the HDs, but 17/20 NMOSD and 11/17 MS samples as well as 13/15 SLE samples had at least 0.7 \% LDGs. NMOSD patients without continuous immunosuppressive treatment had significantly more LDGs compared to their treated 
counterparts. LDG nuclear morphology ranged from segmented to rounded, suggesting a heterogeneity within the group.

Conclusion: LDGs are a feature of the immunophenotype in some patients with MS and NMOSD.

Keywords: low-density granulocytes, multiple sclerosis, neuromyelitis optica, neuroinflammation, autoimmunity

\section{INTRODUCTION}

Low-Density Granulocytes (LDGs) are neutrophilic granulocytes that remain in the fraction of peripheral blood mononuclear cells (PBMC) after density gradient separation. When first described, they were noted to be a feature of rheumatologic diseases such as Systemic Lupus Erythematosus (SLE) (1), while more recent works described LDGs in various conditions such as asthma (2), tuberculosis (3), and psoriasis (4). LDGs have been implicated in the pathogenesis of SLE by producing type I interferons and undergoing spontaneous NETosis (generation of Neutrophil extracellular traps), thus providing potentially immunogenic nuclear matter $(5,6)$. Their role in the pathogenesis of other diseases is less clear.

The origin of the LDGs has not yet been elucidated and it has been proposed that they represent immature or degranulated neutrophils. Interestingly, LDGs in SLE display an immature nuclear structure even though they express the surface markers of mature granulocytes, such as CD16 $(5,6)$.

Neuromyelitis Optica Spectrum Disorder (NMOSD) and Multiple Sclerosis (MS) are neuroinflammatory conditions of unknown etiology. The evidence for the involvement of granulocytes in the pathogenesis of MS has been scarce so far, while some early studies indicate a role of neutrophils in NMOSD pathogenesis $(7,8)$.

In this study, we provide the first evidence for the existence of LDGs in MS and NMOSD and thus add another puzzle piece to the role of the innate immune system in the pathogenesis of these diseases.

\section{MATERIALS AND METHODS}

SLE patients were recruited from the rheumatologic outpatient departments at Charité Universitätsmedizin Berlin. Data on MS- and NMOSD-patients were derived from ongoing observational studies at the NeuroCure Clinical Research Center, Charité-Universitätsmedizin Berlin without prior selection according to sex, age, or other clinical parameters. These studies were approved by the ethics committee of the Charité-Universitätsmedizin Berlin (MS: EA1/163/12; NMOSD: EA1/041/14) and conducted according to the 1964 Declaration of Helsinki in its currently applicable version. Written, informed consent was obtained from all participants in the study.

MS-diagnosis was made according to the 2010 revised McDonald criteria (9), NMOSD-diagnosis was made according to the international consensus diagnostic criteria for NMOSD
2015 (10). All SLE patients fulfilled the 1997 ACR classification criteria (11).

All MS and NMOSD patients were a stable phase of disease with a minimum of 3 months since the last relapse and last glucocorticoid pulse. We excluded patients if they had additional autoimmune, infectious or malignant conditions. We correlated the LDG percentage with clinical information such as Expanded Disability Status Scale (EDSS) score, number of relapses, treatment, and duration of disease as well as titers for anti-MOG (myelin oligodendrocyte glycoprotein) and anti$\mathrm{AQP}-4$ (aquaporin-4) antibodies.

\section{PBMC Isolation, Staining, and Flow Cytometry}

We isolated the PBMC layer with density-gradient separation of $10 \mathrm{ml}$ heparin-anticoagulated blood, using the Ficoll-Paque PLUS gradient (GE Healthcare). PBMCs were washed once in phosphate-buffered saline (PBS) supplemented with 3\% bovine serum albumin (BSA) and subsequently stained with the following antibody-conjugates: CD45 FITC (Miltenyi Biotec Cat\# 130-080-202, RRID:AB_244234), CD14 Cy5 (DRFZ, clone TM1), CD15 PE-Cy7 (BioLegend Cat\# 323029, RRID:AB_2561669), CD16 PE (BioLegend Cat\# 302008, RRID:AB_314208), CD14 APC-Cy7 (BioLegend Cat\# 367108, RRID:AB_2566710), and CD11c Pacific Blue (BioLegend Cat\# 337212, RRID:AB_1595430). We acquired the sample using a FACSCanto (BD Biosciences) or MACSQuant (Miltenyi) cytometer. For imaging flow cytometry (Amnis), we isolated and stained the cells as described above, fixed the sample with the Foxp3 / Transcription Factor Staining Buffer Set (eBioscience) and acquired the cells on an Amnis ImageStream X Mark II. All cytometry experiments were performed in accordance with the "Guidelines for the use of flow cytometry and cell sorting in immunological studies" (12).

\section{FACS Data Analysis}

We excluded sub-cell-sized detritus and doublets based on scatter characteristics and gated on $\mathrm{CD} 45^{+}$leukocytes. Of these, we defined LDGs as $\mathrm{CD} 14^{-} \mathrm{CD} 15^{\text {high }}$ and calculated the ratio of LDGs of all CD45 ${ }^{+}$PBMCs (Figure 1A). We then determined the fraction of CD16 $6^{\text {high }}$ LDGs. FACS Data Analysis was performed using FlowJo Version 10.4.1 (FlowJo LLC). We analyzed the ImageStream data with IDEAS Version 6.2 (Amnis).

\section{Statistical Analysis}

The percentage of LDGs of different disease states and the percentage of CD16 $6^{\text {high }}$ LDGs were compared to HDs using the Kruskal-Wallis test with Dunn's Correction for multiple testing. 


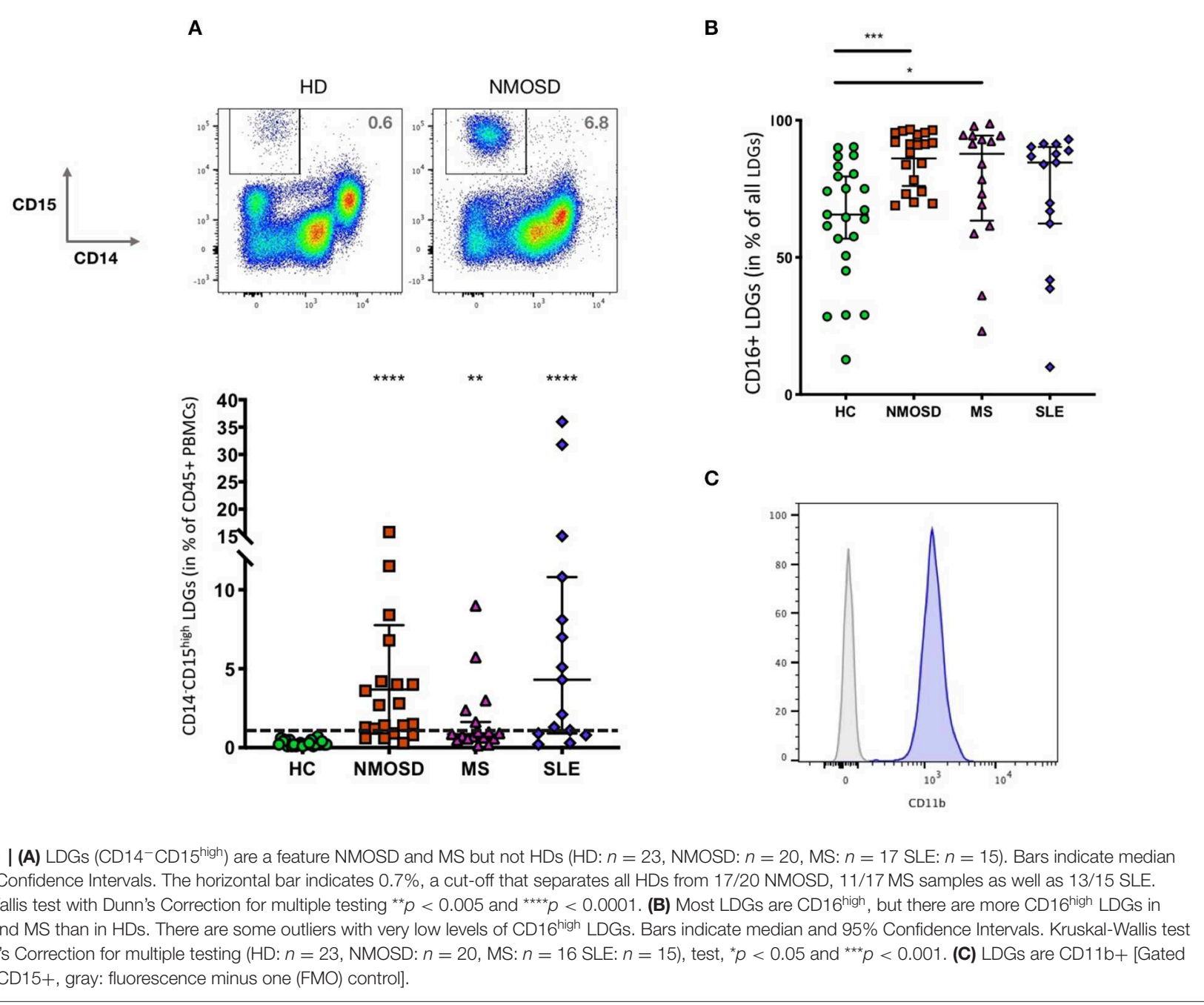

For the difference between two groups, we used the MannWhitney test. All statistical analysis was performed in GraphPad Prism Version 7.0e for Mac OS X (GraphPad Software).

\section{RESULTS}

LDGs are a feature of many inflammatory conditions and of unknown pathogenic significance. To elucidate whether LDGs also exist in NMOSD and MS, we performed FACS analyses of 76 peripheral blood samples.

\section{Cohort Description}

We conducted the analysis of $17 \mathrm{MS}$ patients as well as 20 patients with NMOSD, of which 8 were anti-AQP4 positive and 4 positive for anti-MOG antibodies. Fifteen patients with SLE and 23 HDs served as controls. Median EDSS scores were 4.0 in the MS and 3.0 in the NMOSD group. The most common treatments in the MS group were dimethylfumarate (6/17) and beta Interferon (4/17). The median number of relapses in the relapse-remitting MS (RRMS) patients was 2.5. NMOSD Patients were most often treated with Rituximab (8/20), Mycophenolate $(4 / 20)$, and azathioprine (4/20) (Table 1).

\section{Low-Density Granulocytes Are a Feature of Both Multiple Sclerosis and Neuromyelitis Optica}

In comparison to healthy controls, both MS and NMO patients feature a significantly higher percentage of LDGs. SLE samples displayed the highest frequencies of LDGs (MS: 0.9\%, NMO $2.1 \%$, SLE: $4.3 \%$, HD: $0.2 \%$, median fraction of $\mathrm{CD} 45^{+}$cells) (Figure 1A). None of the HDs, but 17/20 NMOSD and 11/17 MS samples as well as 13/15 SLE samples had at least $0.7 \%$ LDGs. The fraction of CD16 ${ }^{\text {high }}$ LDGs differed both between and within groups (Figure 1B), but was significantly higher in NMOSD and MS compared to HCs. LDGs uniformly expressed CD11b (Figure 1C).

\section{LDGs Show a Heterogeneous Nuclear Morphology}

We analyzed the nuclear morphology of the LDGs of patients with NMOSD, MS, and SLE. While some of the CD14 ${ }^{-}, \mathrm{CD} 15^{\text {high }}$ 
TABLE 1 | Epidemiological information of the study population.

\begin{tabular}{|c|c|c|c|c|}
\hline & NMOSD $(n=20)$ & MS $(n=17)$ & $\mathrm{HD}(n=23)$ & SLE $(n=15)$ \\
\hline Age (median and interquartile range) & $48(34.0-56.5)$ & $47(36.0-51.0)$ & $29(27.0-43.5)$ & $40(31.0-48.0)$ \\
\hline Sex ( $n$ and percentage female) & $17(85.0)$ & $5(29.4)$ & $15(65.2)$ & 14 (93.3) \\
\hline $\begin{array}{l}\text { Time since disease onset (in years, } \\
\text { median and interquartile range) }\end{array}$ & $7.0(3.0-8.8)$ & $9.7(4.6-15.4)$ & - & $13.0(5.5-17.7)$ \\
\hline $\begin{array}{l}\text { Time since last relapse (in years, } \\
\text { median and interquartile range) }\end{array}$ & n.d. & $3.4(1.5-8.3)$ & - & n.d. \\
\hline Treatments ( $n$, percentage) & $\begin{array}{l}\text { Rituximab } n=8(40.0), \\
\text { Azathioprine and untreated } \\
\text { each } n=4(20.0) \text {, } \\
\text { Mycophenolate } n=3(15.0) \text {, } \\
\text { Teriflunomide } n=1 \text { (5.0) }\end{array}$ & $\begin{array}{l}\text { Dimethylfumarat } n=6 \text { (35.3), } \\
\text { Interferon beta } n=4(23.4) \text {, } \\
\text { untreated } n=3(17.6) \\
\text { Daclizumab, intrathecal Steroids, } \\
\text { Glatirameracetate and } \\
\text { Fingolimod each } n=1(5.9)\end{array}$ & - & $\begin{array}{l}\text { Prednisolone } n=15 \text { (100.0), } \\
\text { Hydroxychloroquine } n=7(46.7) \text {, } \\
\text { Mycophenolate } n=3(20.0), \\
\text { Azathioprine, Methotrexate and } \\
\text { Cyclosporine A each } n=2 \\
\text { (13.3), Rituximab } n=1 \text { (6.67) }\end{array}$ \\
\hline $\begin{array}{l}\% \text { of LDGs (median and interquartile } \\
\text { range) }\end{array}$ & $2.1(1.1-4.1)$ & $0.9(0.57-1.63)$ & $0.2(0.2-0.4)$ & $4.3(1.0-9.5)$ \\
\hline $\begin{array}{l}\% \text { of CD16 high LDGs (median and } \\
\text { interquartile range) }\end{array}$ & $91.2(77.2-94.9)$ & $87.8(67.3-94.4)$ & $65.6(53.7-79.9)$ & $84.6(64.6-89.6)$ \\
\hline
\end{tabular}

n.d. no data.

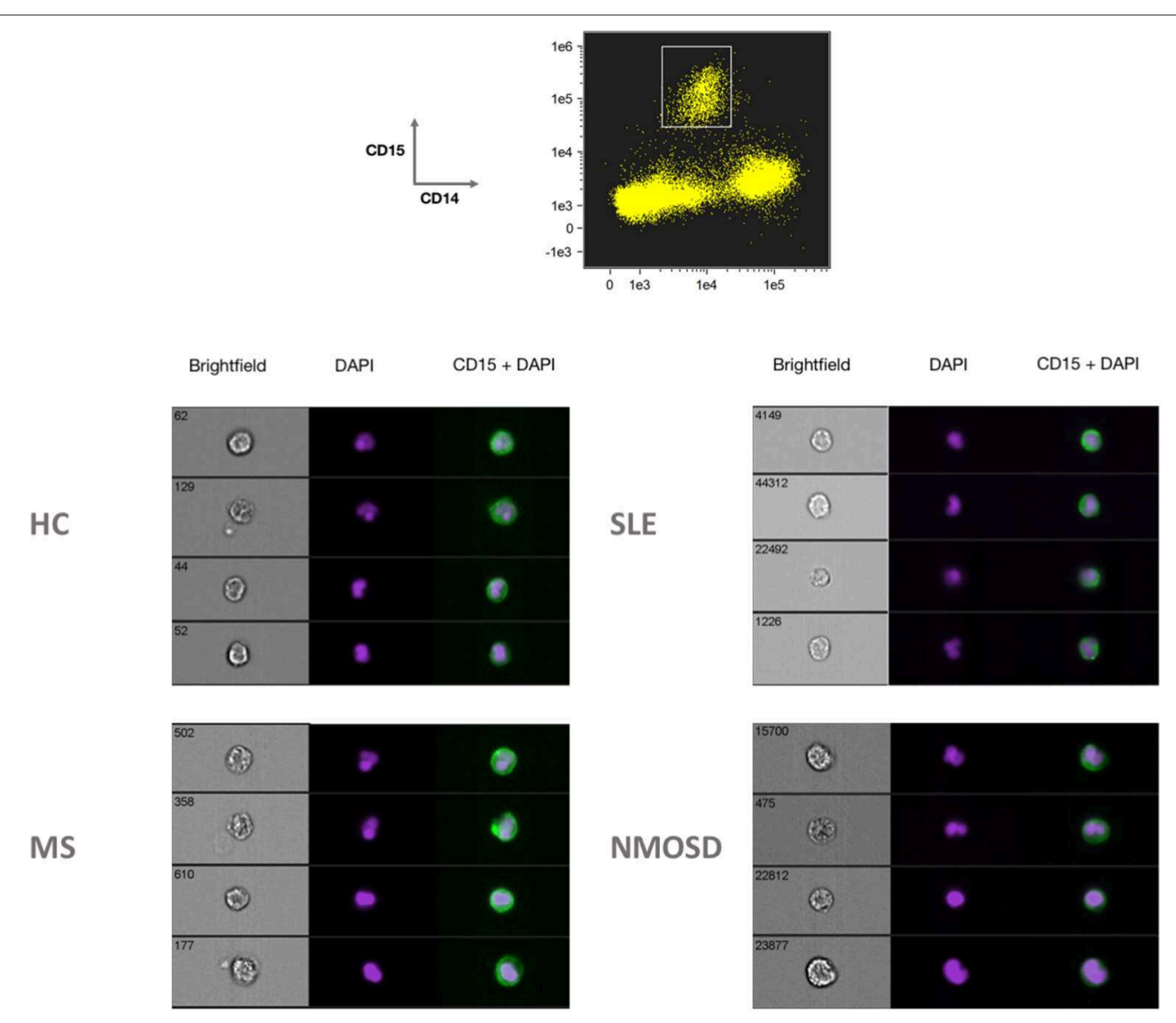

FIGURE 2 | Imaging flow cytometry analysis of LDGs from a healthy donor and patients with SLE, MS, and NMOSD revealed LDGs with heterogenous nuclear shapes, ranging from round to multisegmented. 
cells displayed the segmented nuclear shape typical of mature granulocytes, others showed band-formed or rounded nuclei, which are traditionally thought to be immature forms (Figure 2).

\section{Untreated NMOSD Patients Show Higher LDG Fractions}

We looked for associations between the fraction of LDGs in MS and NMOSD patients and their clinical characteristics such as time since first manifestation of disease, number of relapses, severity of disability as judged by the EDSS score, type of treatment and antibody status, but did not find any significant correlations (data not shown). NMOSD patients who did not receive a continuous immunosuppressive treatment had significantly higher LDG percentages compared to their peers receiving treatment (median LDGs treated 1.4\%, untreated $7.9 \%$, $p<0.01$ ). The percentage of the LDG fraction did not correlate with clinical severity, number of relapses or antibody status.

\section{DISCUSSION}

This study demonstrates the presence of Low-Density Granulocytes in MS and NMOSD.

In contrast to MS, in NMOSD classical granulocytes are thought to play a pivotal role as they can be found in tissue biopsies (7) and alterations in neutrophil function have been described (8). We recently described CD11b+ leucocytes in the PBMCs of MS patients that were characterized by increased activation of $\mathrm{NAD}(\mathrm{P}) \mathrm{H}$ oxidase (NOX) (13). In these, NOX activation correlated with clinical activity. While we assumed these cells to be monocytes at the time, the presence of LDGs in the peripheral blood mononuclear cell (PBMC) fraction could pose an alternative explanation for our previous results.

We found the LDG fraction of NMOSD and MS patients to contain significantly more $\mathrm{CD} 16^{\text {high }}$ cells, a marker indicating neutrophil maturity. As previously described for LDGs in SLE, LDG nuclei in NMOSD vary between mature segmented and immature round forms. In previous studies, the immature nuclear morphology was associated with an expression of surface markers characteristic of mature cells, suggesting that LDGs do not fit into the traditional maturity spectrum of granulocytes (6).

This work adds MS and NMOSD to the diverse list of inflammatory conditions featuring LDGs. In our opinion, this makes it more likely that LDGs are an epiphenomenon of ongoing inflammation rather than a causal part of the pathogenesis of a specific disease, but further research is needed to establish the role of these cells in different conditions. Interestingly, NMOSD patients often have additional immunemediated disorders, especially SLE (14). The fact that these

\section{REFERENCES}

1. Hacbarth E, Kajdacsy-Balla A. Low density neutrophils in patients with systemic lupus erythematosus, rheumatoid arthritis, and acute rheumatic fever. Arthritis Rheum. (1986) 29:1334-42. doi: 10.1002/art.1780291105

2. Fu J, Tobin MC, Thomas LL. Neutrophil-like low-density granulocytes are elevated in patients with moderate to severe persistent asthma. Ann Allergy Asthma Immunol. (2014) 113:635-40.e2. doi: 10.1016/j.anai.2014.08.024 disorders both show high fractions of LDGs could also point toward shared pathogenetic mechanisms. A recent study reported a strong correlation of LDGs and glucocorticoid use in large transcriptional data sets of SLE patients (15). This is in contrast to our results, as none of the MS and NMOSD patients received systemic glucocorticoid treatment in the last 3 months.

To establish the potential of LDGs as a biomarker in neuroinflammatory diseases or inflammation in general, analysis of larger and longitudinal cohorts should be performed to uncover potential correlations with disease severity or prognosis.

\section{DATA AVAILABILITY STATEMENT}

The datasets generated for this study can be obtained from the corresponding author upon reasonable request.

\section{ETHICS STATEMENT}

The studies involving human participants were reviewed and approved by Ethikkommission, Charité - Universitätsmedizin Berlin. The patients/participants provided their written informed consent to participate in this study.

\section{AUTHOR CONTRIBUTIONS}

LO, RM, RN, AH, and HR planed the study and designed the experiments. LO, SK, and RL performed the experiments. JB-S, $\mathrm{SA}, \mathrm{KR}, \mathrm{TA}$, and FP cared for the patients and provided clinical information. LO performed the statistical analysis and wrote the manuscript. HR supervised the work. All authors provided input to the manuscript and approved the final draft.

\section{FUNDING}

This work was supported by DFG TRR130, TPC01 (to RN and $\mathrm{AH}$ ), TP17 (to AH and HR) and by the Leibniz Campus of Chronic Inflammation. LO was member of the Leibniz Graduate School of Chronic Inflammation.

\section{ACKNOWLEDGMENTS}

We thank Peggy Mex (DRFZ) for technical assistance. We are indebted to our patients and healthy controls for their blood donations. We acknowledge support from the German Research Foundation (DFG) and the Open access Publication Fund of Charité - Universitätsmedizin Berlin. A preprint of this manuscript is available at bioRxiv (16).

3. Deng Y, Ye J, Luo Q, Huang Z, Peng Y, Xiong G, et al. Low-density granulocytes are elevated in mycobacterial infection and associated with the severity of tuberculosis. PLoS ONE. (2016) 11:e0153567. doi: 10.1371/journal.pone.0153567

4. Lin AM, Rubin CJ, Khandpur R, Wang JY, Riblett M, Yalavarthi S, et al. Mast cells and neutrophils release IL-17 through extracellular trap formation in psoriasis. J Immunol. (2011) 187:490-500. doi: 10.4049/jimmunol.11 00123 
5. Carmona-Rivera C, Kaplan MJ. Low-density granulocytes: a distinct class of neutrophils in systemic autoimmunity. Semin Immunopathol. (2013) 35:45563. doi: 10.1007/s00281-013-0375-7

6. Denny MF, Yalavarthi S, Zhao W, Thacker SG, Anderson M, Sandy AR, et al. A distinct subset of proinflammatory neutrophils isolated from patients with systemic lupus erythematosus induces vascular damage and synthesizes type I IFNs. J Immunol. (2010) 184:3284-97. doi: 10.4049/jimmunol.090 2199

7. Lucchinetti CF. A role for humoral mechanisms in the pathogenesis of Devic's neuromyelitis optica. Brain. (2002) 125:1450-61. doi: 10.1093/brain/awf151

8. Hertwig L, Pache F, Romero-Suarez S, Stürner KH, Borisow N, Behrens J, et al. Distinct functionality of neutrophils in multiple sclerosis and neuromyelitis optica. Mult Scler J. (2016) 22:160-73. doi: 10.1177/135245851558 6084

9. Polman CH, Reingold SC, Banwell B, Clanet M, Cohen JA, Filippi M, et al. Diagnostic criteria for multiple sclerosis: 2010 revisions to the McDonald criteria. Ann Neurol. (2011) 69:292-302. doi: 10.1002/ana.22366

10. Wingerchuk DM, Banwell B, Bennett JL, Cabre P, Carroll W, Chitnis $\mathrm{T}$, et al. International consensus diagnostic criteria for neuromyelitis optica spectrum disorders. Neurology. (2015) 85:177-89. doi: 10.1212/WNL.0000000000001729

11. Hochberg MC. Updating the American College of Rheumatology revised criteria for the classification of systemic lupus erythematosus. Arthritis Rheum. (1997) 40:1725. doi: 10.1002/art.1780400928

12. Cossarizza A, Chang HD, Radbruch A, Akdis M, Andrä I, Annunziato F, et al. Guidelines for the use of flow cytometry and cell sorting in immunological studies. Eur J Immunol. (2017) 47:1584-797. doi: 10.1002/eji.201646632

13. Mossakowski AA, Pohlan J, Bremer D, Lindquist R, Millward JM, Bock M, et al. Tracking CNS and systemic sources of oxidative stress during the course of chronic neuroinflammation. Acta Neuropathol. (2015) 130:799-814. doi: 10.1007/s00401-015-1497-x

14. Jarius S, Ruprecht K, Wildemann B, Kuempfel T, Ringelstein M, Geis C, et al. Contrasting disease patterns in seropositive and seronegative neuromyelitis optica: a multicentre study of 175 patients. J Neuroinflammation. (2012) 9:14. doi: 10.1186/1742-2094-9-14

15. Kegerreis BJ, Catalina MD, Geraci NS, Bachali P, Lipsky PE, Grammer AC. Genomic identification of low-density granulocytes and analysis of their role in the pathogenesis of systemic lupus erythematosus. J Immunol. (2019) 202:3309-17. doi: 10.4049/jimmunol.1801512

16. Ostendorf P, Mothes P, van Koppen S, Lindquist PL, Bellmann-Strobl J, Asseyer S, et al. Low-density granulocytes are a novel immunopathological feature in both Multiple Sclerosis and Neuromyelitis optica spectrum disorder. bioRxiv. doi: 10.1101/668160v1

Conflict of Interest: KR received research support from Novartis, Merck Serono, and German Ministry of Education and Research as well as speaking fees and travel grants from Bayer Healthcare, Biogen Idec, Merck Serono, sanofiaventis/Genzyme, Teva Pharmaceuticals, Roche, Novartis, and Guthy Jackson Charitable Foundation. FP served on the scientific advisory boards of Novartis and MedImmune; received travel funding and/or speaker honoraria from Bayer, Novartis, Biogen, Teva, Sanofi-Aventis/Genzyme, Merck Serono, Alexion, Chugai, MedImmune, and Shire; is an associate editor of Neurology: Neuroimmunology and Neuroinflammation; is an academic editor of PLOS ONE; consulted for Sanofi Genzyme, Biogen, MedImmune, Shire, and Alexion; received research support from Bayer, Novartis, Biogen, Teva, Sanofi-Aventis/Geynzme, Alexion, and Merck Serono; and received research support from the German Research Council, Werth Stiftung of the City of Cologne, German Ministry of Education and Research, Arthur Arnstein Stiftung Berlin, EU FP7 Framework Program, Arthur Arnstein Foundation Berlin, Guthy-Jackson Charitable Foundation, and NMSS. HR received speaker honoraria and research support from Novartis and Sanofi-Aventis/Genzyme unrelated to this project.

The remaining authors declare that the research was conducted in the absence of any commercial or financial relationships that could be construed as a potential conflict of interest.

Copyright (c) 2019 Ostendorf, Mothes, van Koppen, Lindquist, Bellmann-Strobl, Asseyer, Ruprecht, Alexander, Niesner, Hauser, Paul and Radbruch. This is an openaccess article distributed under the terms of the Creative Commons Attribution License (CC BY). The use, distribution or reproduction in other forums is permitted, provided the original author(s) and the copyright owner(s) are credited and that the original publication in this journal is cited, in accordance with accepted academic practice. No use, distribution or reproduction is permitted which does not comply with these terms. 